\title{
Versorgungsmöglichkeiten am distalen Femur und Femurschaft: der Vorteil der Winkelstabilität
}

\author{
Marie K. Reumann, Michael Schütz
}

\section{Zusammenfassung}

Die Therapie von Femurschaft- und distalen Femurfrakturen stellt auch heute noch oftmals eine chirurgische Herausforderung dar, und war lange Zeit mit einer beträchtlichen Komplikationsrate verbunden. Trotz verbesserter Implantate und Techniken waren die Plattenosteosynthese und auch die Marknagelosteosynthese mit z.T. erheblichen Raten an Infektionen, Pseudarthrosen und Fehlstellungen behaftet. Die Beachtung der Weichteilsituation durch „biologische“ Osteosynthesen und minimalinvasive $\mathrm{Zu}$ gänge verringerten die Komplikationsraten deutlich. Aus diesen Überlegungen heraus entstanden die winkelstabilen Plattensysteme wie z. B. das LISS (Less Invasive Stabilization System), deren Vorteile in der winkelstabilen Verankerung der Schrauben, der anatomisch gut angepassten Plattenform und technischen Hilfen zur minimalinvasiven, schonenden Plattenfixation liegen. Dieser Artikel gibt einen kurzen Überblick über die momentan verfügbaren Versorgungsmöglichkeiten distaler Femurfrakturen und Femurschaftfrakturen, mit einem besonderen Schwerpunkt auf den winkelstabilen Systemen. Hierzu ausgewählte Fallbeispiele demonstrieren die Überlegenheit dieser Art der operativen Versorgung besonders bei der Anwendung komplexer distaler Femurfrakturen.

\section{Treatment Options for the Femur Shaft and Distal Femur - Advantages of Angular Stability}

Even today the treatment of fractures of the femoral shaft and distal femur often presents a surgical challenge and was associated with a considerable incidence of complications for a long time. In spite of improved implants and techniques not only plate fixation but also intramedullary nailing were accompanied with significant rates of infection, non-union, and malpositioning. Taking into account the soft-tissue situation by means of "biological fixation" and minimally invasive approaches has markedly reduced the incidence of complications. Based on these considerations, as just one example, the LISS (less invasive stabilisation system) was introduced in the 1990s; advantages of the LISS are the angle-stable fixation of the screws, the anatomically well-adapted plate form and the technical aids for minimally invasive, conserving plate fixation. The present article provides a brief overview of the currently available treatment options for distal femur and femoral shaft fractures with particular emphasis on angle-stable systems. Selected case reports demonstrate the superiority of this method for surgical treatment, especially when used for complex distal femur fractures.

\section{Einleitung}

Distale Femurfrakturen und Femurschaftfrakturen entstehen einerseits durch Hochenergietraumata beim jungen

OP-JOURNAL 2012; 28: 136-142

(C) Georg Thieme Verlag KG Stuttgart · New York DOI http://dx.doi.org/10.1055/s-0032-1315344
Patienten [1,2]. Eine axiale Stauchung, wie sie beim Einklemmen bei Verkehrsunfällen und bei sog „dash board injuries“ vorkommen kann, stellt dabei ein typisches Verletzungsmuster dar („floating knee injury“). Andererseits sind auch Niedrigenergietraumata die Ursache knöcherner Verletzungen am Femur. Hier stehen besonders Frakturen aufgrund schlechter Knochenqualität wie
Osteoporose [3,4] sowie periprothetische Frakturen im Vordergrund, die eine besondere Herausforderung hinsichtlich der Implantatverankerung darstellen.

Die Therapiekonzepte sind vielseitig und reichen von konservativer über operative Therapie, wie extra- und intramedulläre knöcherne Versorgung, bis hin zum primären alloplastischen Gelenkersatz [5-7]. Die Ergebnisse bei der Versorgung besonders von distalen Femurfrakturen waren in den letzten Jahrzehnten mit einer nicht geringen Zahl von Infektionen, Pseudarthrosen und Fehlstellungen belastet [8-10]. Aufgrund dieser Komplikationen wurde bis in die 70er-Jahre von einer routinemäßigen operativen Versorgung abgeraten $[9,11]$. Erst mit der Entwicklung geeigneter Implantate mit guten operativen Ergebnissen wandelte sich die Behandlung hin zur operativen Therapie [12-14].

Im Laufe der folgenden Jahre revolutionierte die Entwicklung neuer Implantatmaterialien, Repositionstechniken, Techniken der Implantatplatzierung und der Implantate selbst die operativen Versorgungsmöglichkeiten. Speziell im Bereich der extramedullären Osteosynthese vollzogen sich mit Einführung der Winkelstabilität maßgebliche Veränderungen.

\section{Versorgungsmöglichkeiten distaler Femurfrakturen}

Ziel der Behandlung distaler Femurfrakturen ist die Wiederherstellung der kondylären Gelenkfläche, anatomischer Achsverhältnisse und einer korrekten Rotation.

Noch in den 80er-Jahren war die operative Versorgung durch weit offene Verfahren und Implantate seinerzeit mit häufigen Komplikationen wie hoher Infektrate, verzögerter Frakturheilung, Implantatversagen oder Retentionsverlust verbunden. 

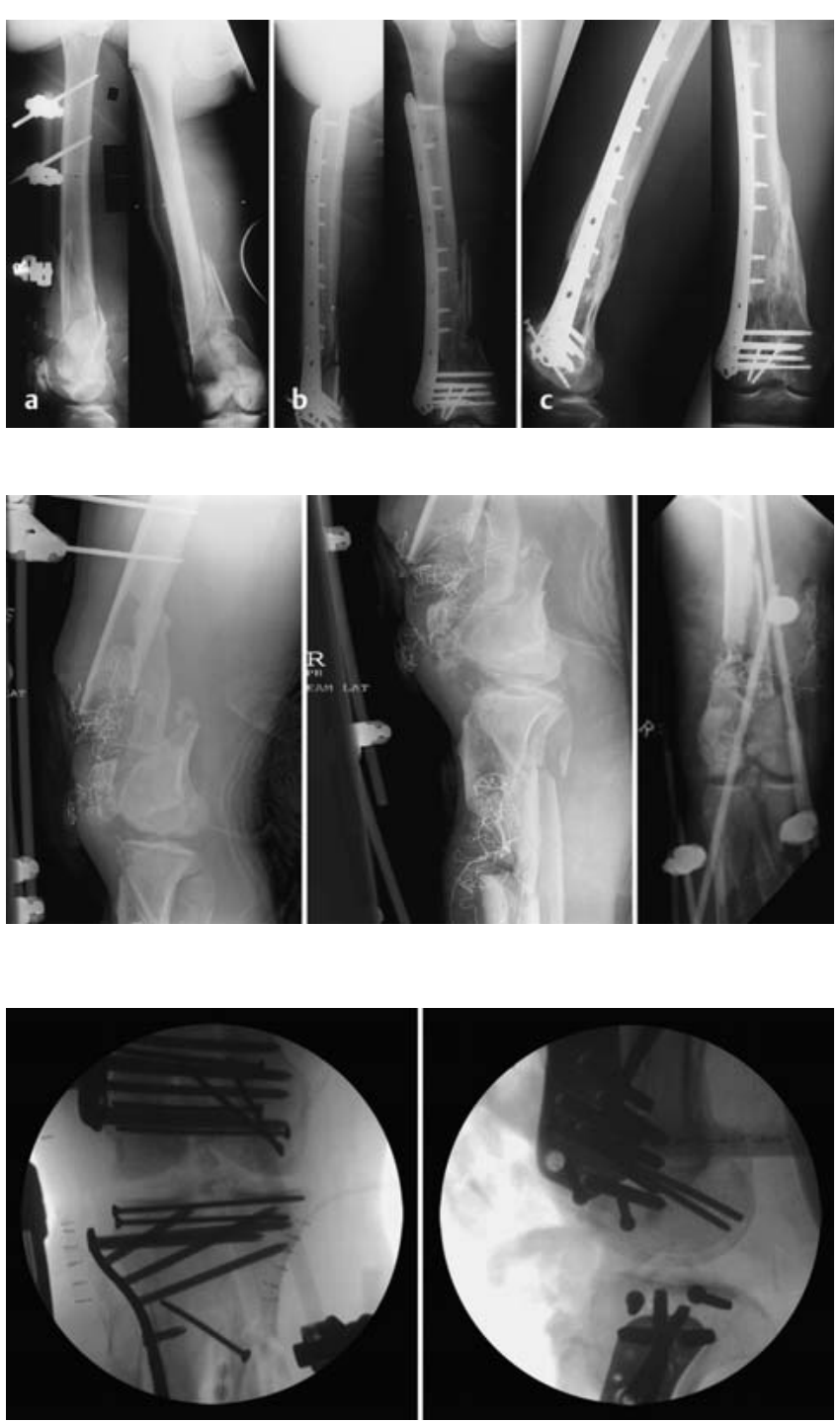

Implantate, die zur Anwendung am distalen Femur kamen, waren die Kondylenplatte („Burri-Platte“) und die DCSPlatte (dynamische Condylenschrauben). Die DCS wies im gewissen Sinne bereits eine Winkelstabilität auf, war aber nicht immer leicht in korrekter Position zu implantieren - was zu einem primären Repositionsverlust führen konnte. Die Kondylenplatte ist ohne jegliche Winkelstabilität der Schrauben verankert und kam bei komplexen intraartikulären Frakturen (C3-Frakturen) zur Anwendung. Nicht selten kam es im Behandlungsverlauf zu einer Lockerung der gelenksnahen Schrauben oder einer Plattenverbiegung aufgrund ungenügender Plattenstärke im metaphysären Bereich mit konsekutivem Repositionsverlust mit Varusfehlstellung.

Mitte der Neunzigerjahre hat dieses Problem zu einer Modifizierung der Implantate und damit verbundener Operations-
Abb. 1 a bis c

(a) Zeigt die präoperativen Aufnahmen der 33-C3-Fraktur nach initialer Stabilisierung mit Fixateur externe. Bild (b) zeigt die postoperative Situation, (c) wurde 34 Monate postoperativ aufgenommen.

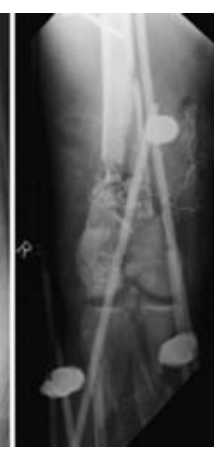

Abb. 3 Zeigt intraoperative Aufnahmen der „floating knee“Verletzung nach Stabilisierung mit winkelstabilen Systemen.

sodass eine frühe Mobilisierung des Kniegelenks erreicht werden kann.

Wie kein anderes Implantat zuvor decken die winkelstabilen Plattensysteme nahezu das ganze Spektrum an Frakturtypen ab und können überdies bei nahezu allen periprothetischen Frakturen zum Einsatz kommen [18, 25].

Im Folgenden zeigen 3 Fallbeispiele die unterschiedlichen Techniken der operativen Versorgung distaler Femurfrakturen. präoperativen Aufnahmen der distalen Femurfraktur sowie der proximalen Tibiafraktur nach initialer Stabilisierung mit Fixateur externe.

Der 1. Patient (männlich, 33 Jahre) erlitt neben multiplen anderen Verletzungen eine zweitgradig offene Fraktur 33-C3 nach AO nach einem Sturz aus großer Höhe. Am Aufnahmetag wurde die offene Fraktur gereinigt, debridiert und mit einem Fixateur externe stabilisiert (Abb.1a). Am 4. Tag nach Aufnahme konnte dann eine definitive Osteosynthese erfolgen (Abb.1 b). Ein 13-LochLISS und 4 Zugschrauben wurden benutzt, zusätzlich wurde die frakturierte Eminentia intercondylaris fixiert und es erfolgte eine Patellektomie. Eine primäre Spongiosaplastik wurde nicht durchgeführt. Bei der letzten Kontrolle (34 Monate postoperativ [Abb.1 c]) war die Fraktur mit einem minimalen ventralen Kortikalisdefekt konsolidiert, wegen dem die Entfernung des Implantats bis zu diesem Zeitpunkt nicht durchgeführt worden war. Der Patient war insgesamt schmerzfrei, die Extension/Flexion betrug $0 / 0 / 120^{\circ}$ und es bestand keine axiale Fehlstellung oder Verkürzung.

technik im Sinne der „biologischen“ Osteosynthese geführt. Aus heutiger Sicht hat hierbei die Winkelstabilität die Versorgung vom distalen Femur revolutioniert. Auch wenn die Evidenz klinischer Studien bisher keinen Vorteil aufzeigen konnte [15-19], so wird heutzutage weltweit der überwiegende Anteil von distalen Femurfrakturen mit vorgeformten, winkelstabilen Platten versorgt. Als erstes Implantat hat das „LISS - Less Invasive Stabilization System“ für eine deutliche Erleichterung der Versorgung komplexer artikulärer und osteoporotischer Frakturen gesorgt. Im Sinne der „biologischen“ Osteosynthese bleiben die Weichteile geschont, bei gleichzeitig geringer Beschädigung der Durchblutung [20-24]. Die vorgeformte Platte erleichtert die Reposition hinsichtlich korrekter Achsausrichtung und Rotation. Weiterhin ermöglicht das LISS eine insgesamt gute Sicherung des Gelenkblocks,
Der 2. Patient (männlich, 35 Jahre) hatte sich im Rahmen ein Motorradunfalls eine drittgradig offene, distale Femurfraktur (IIIB, 33-C3) mit proximaler Tibiafraktur zugezogen (,floating knee injury“). Die initiale Versorgung erfolgte mittels Fixateur externe im auswärtigen Krankenhaus (Abb. 2). In unserer Abteilung wurde die definitive Versorgung mit winkelstabilen Plattensystemen und freien 3,5-mm-Zugschrauben durchgeführt (Abb. 3 und 4).

Die anatomisch vorgeformten Platten führen zu einer deutlichen Erleichterung der achsgerechten Ausrichtung.

Die Weichteildeckung erfolgte am 5. Tag mittels eines Latissimus-dorsi-Lappens. Drei Monate nach Unfall wurde außerdem ein Knochenersatz mit Spongiosa an der proximalen Tibia durchgeführt. Eine Nachuntersuchung nach 1 Jahr zeig- 


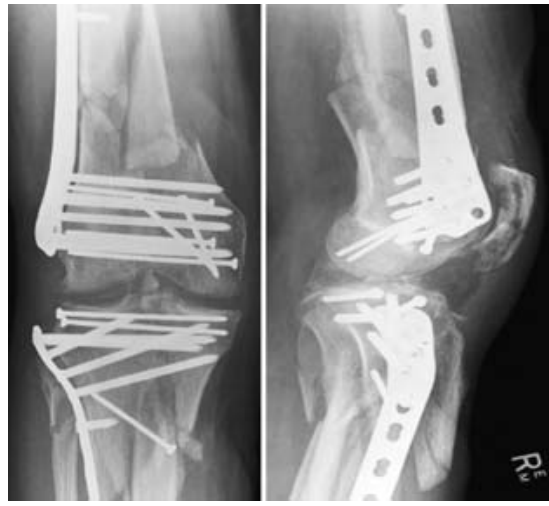

Abb. 4 Nachuntersuchung 6 Wochen postoperativ.

te eine insgesamt komplikationslose Ausheilung mit knöcherner Konsolidierung an distalem Femur und proximaler Tibia bei gutem Bewegungsumfang des Kniegelenks von 0/0/120 (Abb. 5).

Der 3. Fall zeigt die Verletzung einer 42jährigen PKW-Fahrerin, die im Rahmen eines Verkehrsunfalls eine „floating knee“-Verletzung mit drittgradig offener distaler Femurfraktur mit Knochenverlust erlitten hatte. Die initiale Versorgung war im auswärtigen Krankenhaus mit retrograder Marknagelosteosynthese erfolgt (Abb. 6). Sechs Wochen postoperativ war uns die Patientin wegen anhaltender Schmerzen und Lockerung der distalen Schrauben zuverlegt worden (Abb. 7). In unserem Haus erfolgte daraufhin die Implantatentfernung (Abb. 8) und Restabilisierung mit LISS-Implantat und gleichzeitiger Anschraubung kortikospongiöser Spänen vom Beckenkamm zur Auffüllung des Knochendefekts (Abb. 9). Nach weiteren 6 Monaten wurde eine nochmalige Knochenanlagerung im Schaftbereich durchgeführt (Abb. 10). Insgesamt $1 \mathrm{Jahr}$ nach operativer Versorgung in unserem Haus war eine stabile Ausheilung der Verletzung mit guter Funktion (siehe Abb.11) erreicht worden.

\section{Versorgungsmöglichkeiten von Frakturen am Femurschaft}

Wie auch bei der Behandlung der distalen Femurfrakturen steht am Femurschaft primär als Ziel die Wiederherstellung der korrekten Achsverhältnisse, Rotation sowie der korrekten Beinlänge im Vordergrund.

Die Implantatwahl am Femurschaft hängt von mehreren Faktoren ab. Nicht nur Frakturtyp, -lokalisation und Weichteilsituation spielen eine entscheidende

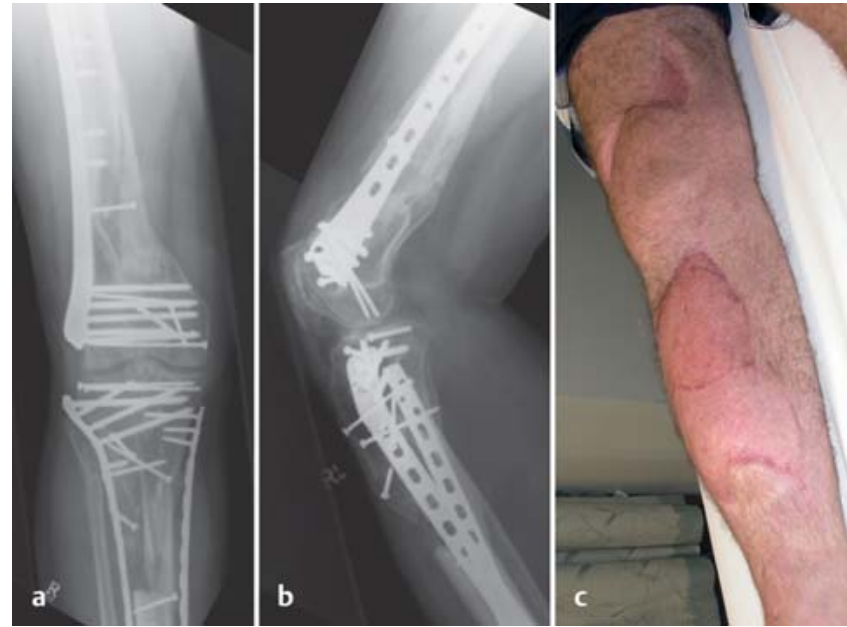

Abb. 5 a bis c

Nachuntersuchung nach 1 Jahr postoperativ. Bilder (a) und (b) zeigen die radiologischen Aufnahmen der knöchern konsolidierten Frakturen. Bild (c) zeigt die ausgeheilten Weichteilverhältnisse der rechten unteren Extremität nach Lappenplastik.
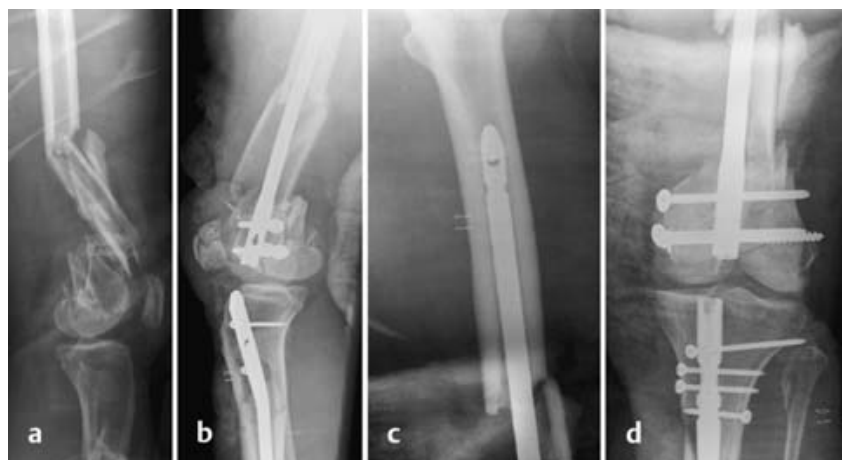

Abb. 6 a bis d

Bild (a) zeigt das initiale "floating knee“Verletzungsmuster mit drittgradig offener 33-C3 mit Knochenverlust sowie zweitgradig offener 42-B1-Fraktur. Bilder (b), (c) und (d) zeigen die radiologischen Aufnahmen postoperativ nach Versorgung in externem Krankenhaus mittels Marknagelung.

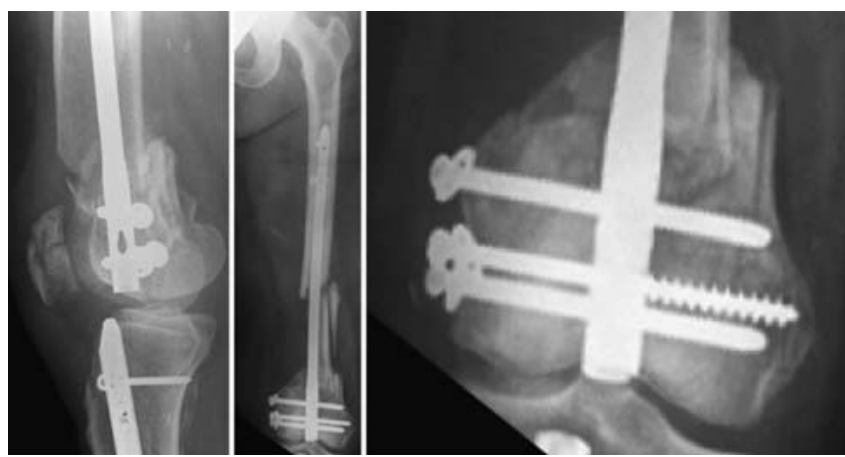

Abb. 7 Röntgenaufnahmen 6 Wochen postoperativ bei Aufnahme in unsere $\mathrm{Kli}$ nik zeigen die fehlende knöcherne Konsolidierung und die Auslockerung der distalen Schrauben.

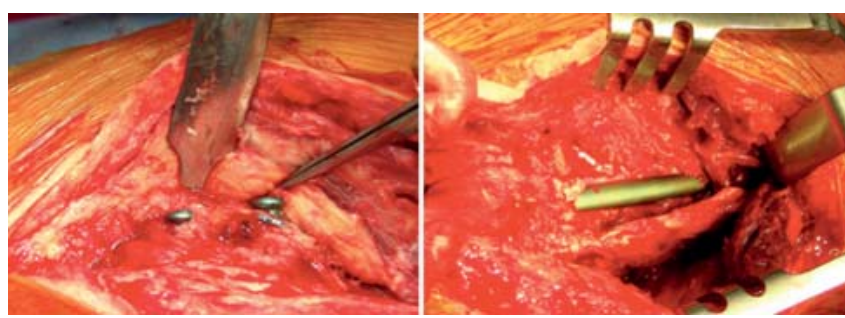

Abb. $8 \mathrm{a}$ und $\mathbf{b}$ Intraoperativer Befund der ausgelockerten Schrauben (a) und des über lange Distanz freiliegenden Marknagels (b).

Rolle, auch bereits in situ vorhandene Implantate, wie im Markraum liegende Prothesen, müssen in Betracht gezogen werden. Weiterhin entscheidend bei der Implantatwahl ist der Allgemeinzustand des Patienten (ISS, Polytrauma, komple- xe Mitverletzungen wie z.B. Beckenringoder Azetabulumfrakturen). Auch wenn die Marknagelung in der Regel die Methode der Wahl am Femurschaft darstellt, ist sie bei mehrfach verletzten Patienten als kritisch zu betrachten auf- 


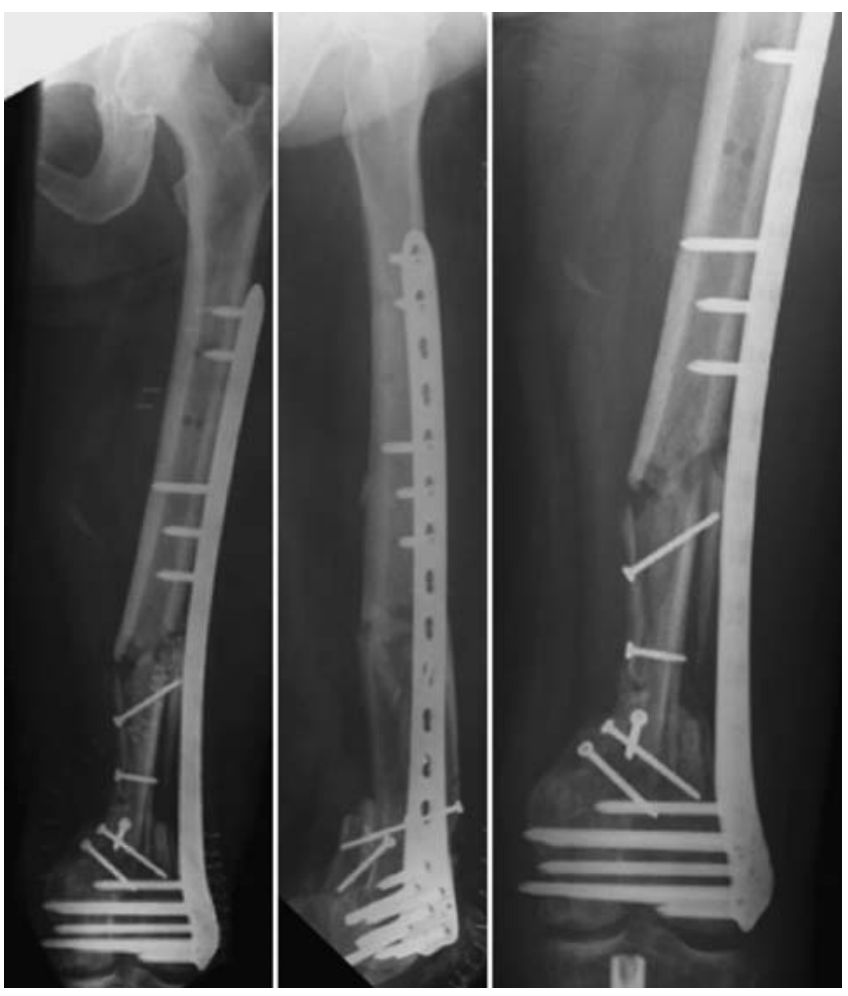

Abb. 9 Postoperativer Röntgenbefund zeigt die nun winkelstabile Versorgung nach Verfahrenswechsel mit zusätzlicher Anschraubung von kortikospongiösen Spänen zur Auffüllung des Knochendefekts.

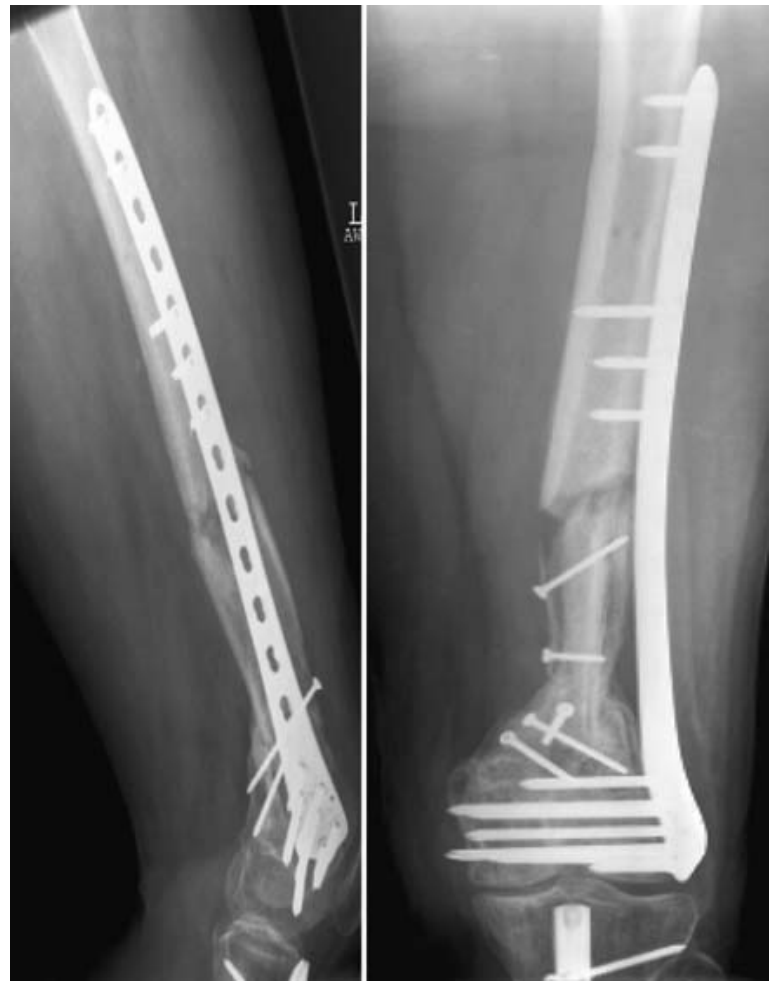

Abb. 10 Nachuntersuchung nach 26 Wochen.

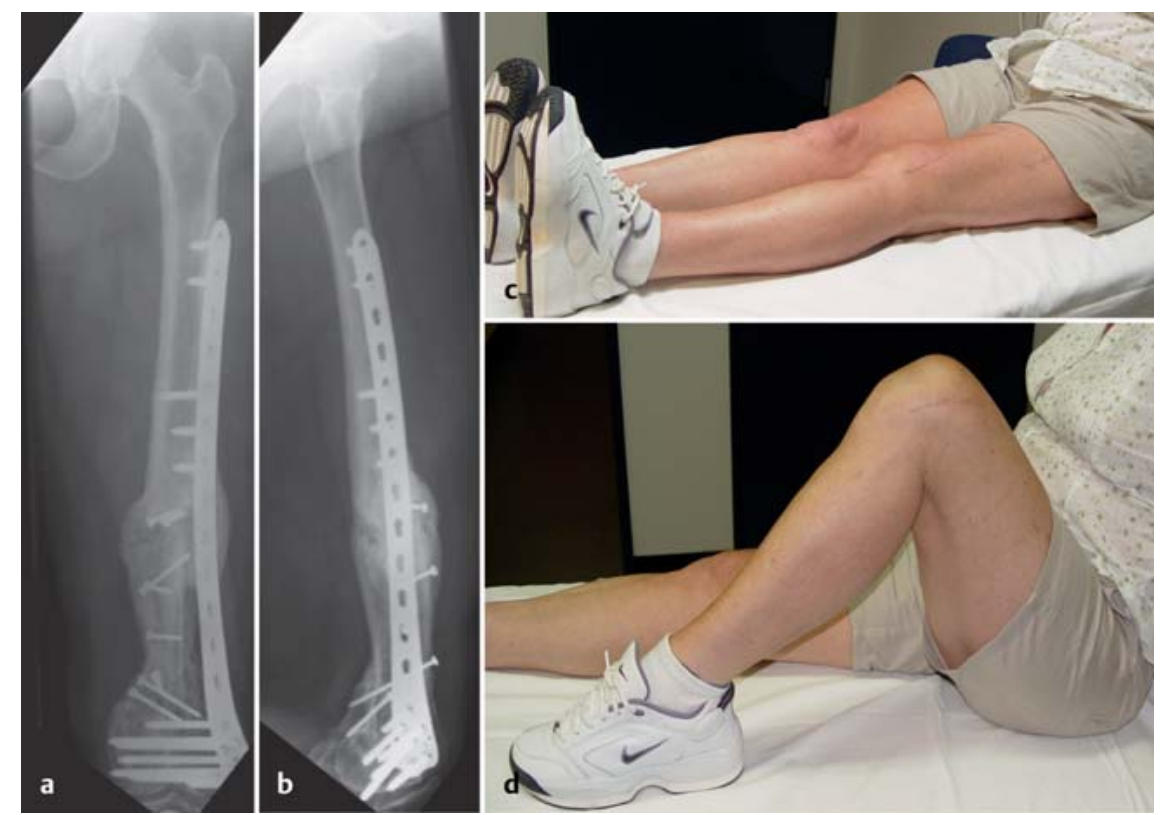

Abb. 11 a bis $\mathbf{d}$ Zeigt den radiologischen (a, b) sowie den klinischen (c, d) Befund 1 Jahr postoperativ.

grund des Risikos von Fettembolien, die beim Einbringen des Marknagels in den Schaft entstehen können [26].

Der Vorteil der Marknagelosteosynthese liegt in der geschlossenen und somit minimalstinvasiven operativen Versor- gungsmöglichkeit, die in der Regel eine hohe Stabilität gewährleistet. Die Versorgung mit winkelstabilen Systemen im Femurschaftbereich ist hingegen seltener. Sie wird dann in Erwägung gezogen, wenn die Marknagelung aufgrund kritischen Allgemeinzustands des Patienten
(Zustand nach Polytrauma, ISS, komplexe Mehrfachverletzungen wie Beckenring- oder Azetabulumfrakturen) oder mangelnder technischer Möglichkeit des Einbringens eines Nagels im Markraum, z.B. bei bereits einliegender Prothese, nicht durchgeführt werden sollte oder kann.

Idealerweise kann in solchen Situationen die Locking Compression Plate (LCP) eingesetzt werden. Sie stellt eine Kombination aus herkömmlichen konventionellen Plattensystemen und den neuen winkelstabilen Fixateur-interne-Systeme dar mit dem Vorteil, im gleichen Plattenloch entweder eine winkelstabile oder eine konventionelle (Kompressions-)Schraube einsetzen zu können.

Zwei ausgewählte Fallbeispiele aus unserer Klinik erläutern die operativen Versorgungsmöglichkeiten am Femurschaft.

Der 1. Fall beschreibt die Situation eines 21-jährigen polytraumatisierten Patienten mit beidseitigen Femurschaftfrakturen und u.a. komplexer Beckenringfraktur. Die initiale Versorgung erfolgte im auswärtigen Krankenhaus mittels Stabilisierung mit Fixateur externe (Abb. 12). Nach der Zuverlegung in unser Haus zur Weiterbehandlung wurde zunächst der 


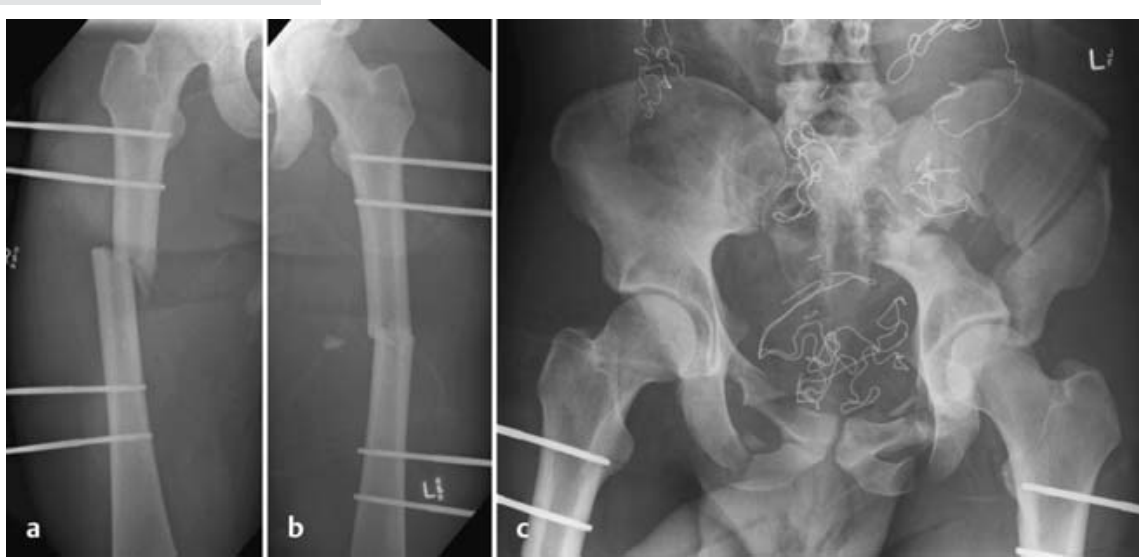

Abb. 12 Zeigt die initiale Verletzung mit (a) rechter und (b) linker Femurschaftfraktur sowie (c) komplexer Beckenringfraktur.
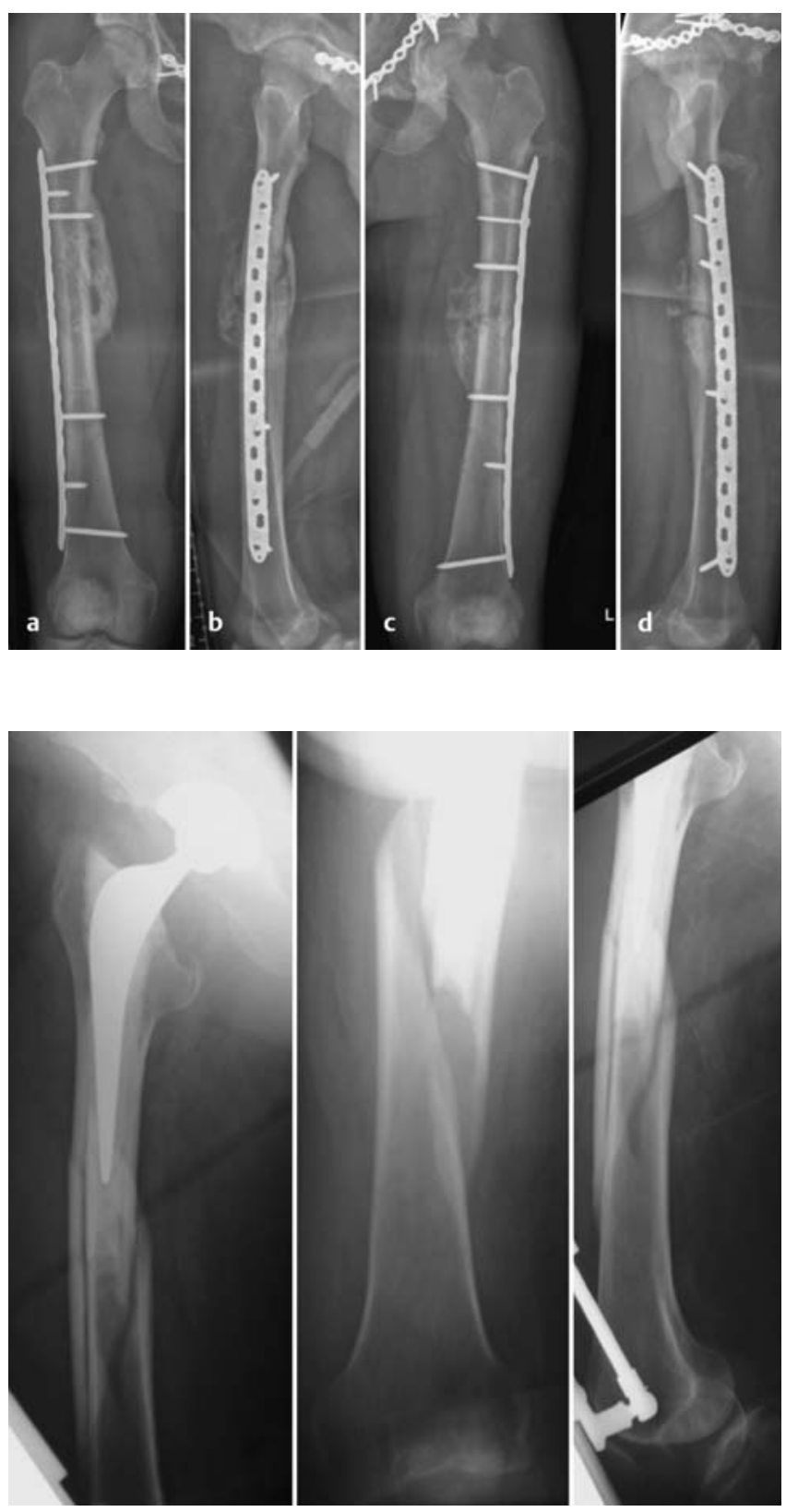

Abb. 14a bis d

Nachuntersuchung 6 Monate postoperativ. Sowohl der rechte (a a.-p. Aufnahme, b seitliche Aufnahme) als auch der linke (c a.-p. Aufnahme, d seitliche Aufnahme) Femurschaft sind bereits knöchern konsolidiert und zeigen deutliche Kallusbildung.

Abb. 15 Initiale knöcherne Verletzung des Femurschafts unterhalb der einliegenden Hüftprothese.

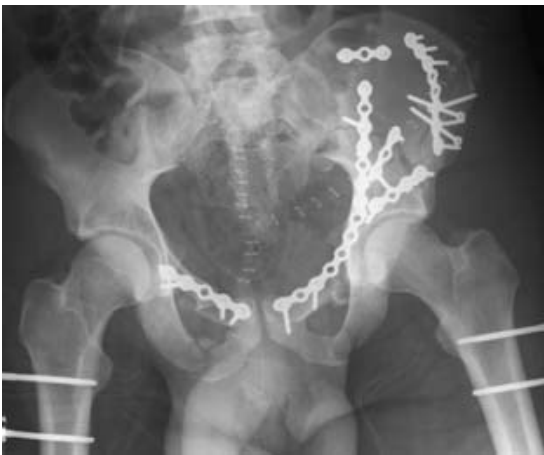

Abb. 13 Zeigt die primäre operative Versorgung der Beckenringfraktur.

Beckenring stabilisiert (Abb. 13). Aufgrund der begleitenden Umstände der Mehrfachverletzung (beidseitige Femurfrakturen, Lungenkontusion/Hämatopneumothorax und Komplexität der Beckenringverletzung) wurde entschieden, eine überbrückende eingeschobene Plattenosteosynthese mit $4,5 \mathrm{~mm}$ breiter, gekrümmter LCP durchzuführen. Bereits nach 6 Monaten waren beide Femurfrakturen mit Kallusbildung ausgeheilt (Abb. 14).

Der 2. Fall beschreibt die Situation eines 79-jährigen Patienten mit periprothetischer Femurschaftfraktur (Abb. 15). Die operative Versorgung erfolgte bei einliegender Prothese mit winkelstabiler Plattenosteosynthese. Als zusätzliche Stabilisierung erfolgte die Verankerung mit "locking attachment plates" um den Schaft (Abb. 16 und 17). In der Nachuntersuchung bereits 4 Monate postoperativ zeigte sich eine gute Ausheilung der Fraktur mit Kallusbildung (Abb. 18).

\section{Nachbehandlung}

Ein weiteres Ziel der Versorgung sowohl bei Femurschaft- als auch bei distalen Femurfrakturen ist es, zu gewährleisten, dass eine frühe Mobilisierung der verletzten Extremität und Physiotherapie erfolgen kann. Ab dem 1. postoperativen Tag beginnt die Bewegungstherapie mithilfe von CPM (continuous passive motion), um Kontrakturen und verbleibende Bewegungseinschränkungen im Kniegelenk zu vermeiden und Schwellungen vorzubeugen. Sofern es der Zustand des Patienten erlaubt, kann eine frühe Mobilisierung an Unterarmgehstützen erfolgen mit Fußsohlenbodenkontakt. Die Belastungssteigerung orientiert sich am Frakturtyp, der erreichten Reposition und Fixation sowie regelmäßigen radiologischen Kontrollen. 

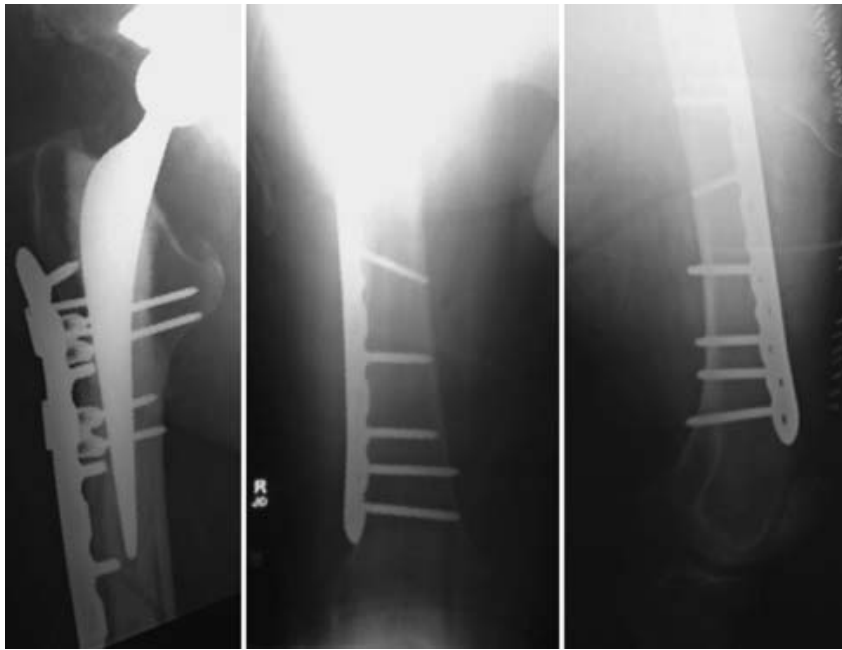

Abb. 16 Operative Versorgung mittels winkelstabilem Plattensystem und zusätzlichen „locking attachment plates“.

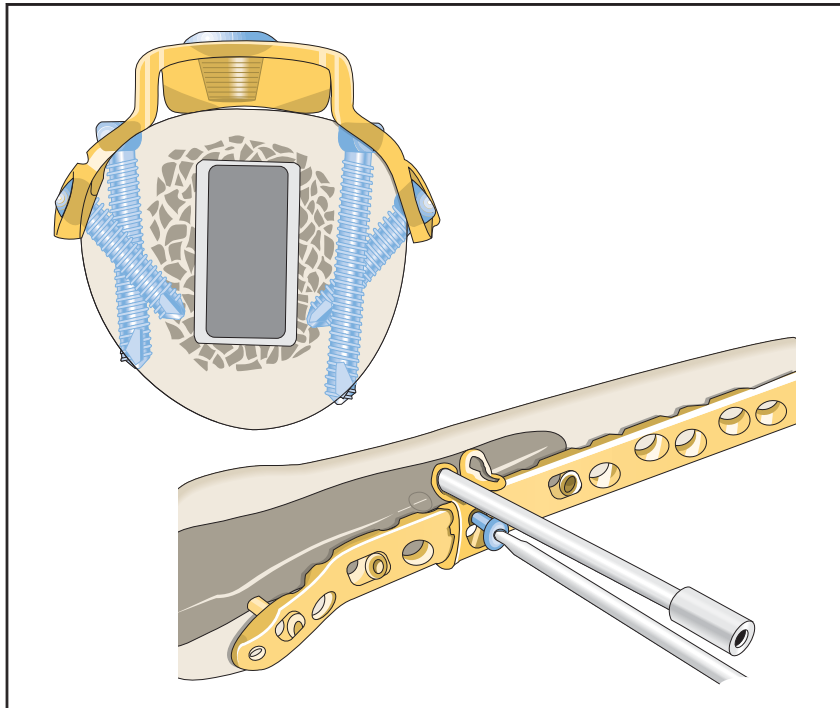

Abb. 17 Technik der „locking attachment plate“ als zusätzliche Verankerungsmöglichkeit im Femurschaft.

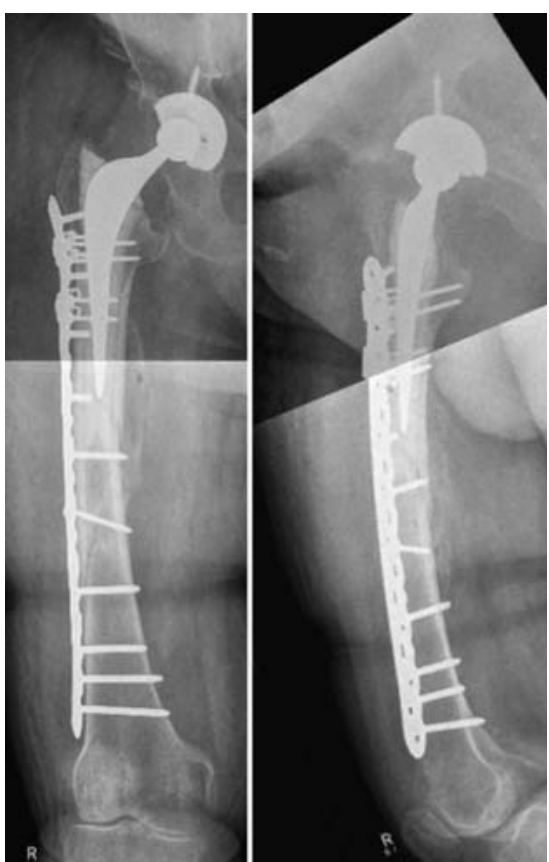

Abb. 18 Die Nachuntersuchung 4 Monate postoperativ zeigt die knöcherne Durchbauung der Femurschaftfraktur mit Kallusbildung.

\section{Zusammenfassung}

In den letzten Jahren haben sich mit den minimalinvasiven Techniken und dem Konzept von winkelstabilen Plattensystemen entscheidende Entwicklungen in der operativen Versorgung distaler Femurfrakturen und Femurschaftfrakturen vollzogen. Winkelstabile Systeme sind durch ihre Konstruktion in der Lage, Weichteile und Durchblutung in idealer Weise zu schonen und erlauben dadurch die „biologische“ Osteosynthese. Durch die Möglichkeit der Winkelstabilität, die sich in den letzten Jahren weltweit als Therapie der Wahl durchgesetzt hat, kann eine Auflage des Kraftträgers auf dem Periost reduziert oder sogar darauf verzichtet und damit die periostale Durchblutung geschont werden. Ein zusätzlicher großer Vorteil der winkelstabilen Systeme liegt in ihrem breiten Indikationsspektrum. Im Bereich des Femurschafts sind minimalinvasive Techniken mit winkelstabilen Systemen im wirklichen Sinne eines Fixateur interne v.a. dann anzuwenden, wenn die Marknagelung kritisch oder nicht durchführbar ist wie bei Polytraumapatienten, Mehrfachverletzungen oder bereits einliegenden intramedullären Implantaten wie Prothesen. Nahezu alle Frakturen des distalen Femurs können winkelstabil versorgt werden: dies reicht von den distalen periprothetischen Femurfrakturen und osteoporotischen Frakturen über die distalen Femurschaftfrakturen und die extraartikulären distalen Femurfrakturen bis hin zu den komplexen intraartikulären Trümmerfrakturen mit metaphysärem Substanzverlust und ausgedehnter Trümmerzone.

\section{Literatur}

${ }^{1}$ Funovics PT, Vecsei V, Wozasek GE. Mid- to long-term clinical findings in nailing of distal femoral fractures. J Surg Orthop Adv 2003; 12: $218-224$

2 Jawadi $A H$, Letts $M$. Injuries associated with fracture of the femur secondary to motor vehicle accidents in children. Am J Orthop (Belle Mead NJ) 2003; 32: 459-462

3 Bengner $U$, Ekbom T, Johnell $O$ et al. Incidence of femoral and tibial shaft fractures. Epidemi- ology 1950-1983 in Malmo, Sweden. Acta Orthop Scand 1990; 61: 251-254

${ }^{4}$ Tejwani NC, Guerado E. Improving fixation of the osteoporotic fracture: the role of locked plating. J Orthop Trauma 2011; 25 (Suppl. 2): S56-S60

${ }^{5}$ Krettek C, Schandelmaier P, Lobenhoffer P et al. [Complex trauma of the knee joint. Diagnosis - management - therapeutic principles]. Unfallchirurg 1996; 99: 616-627

${ }^{6}$ Ruter A, Kotter A. [Fractures of the distal femur]. Unfallchirurg 1996; 99: 510-519

${ }^{7}$ Sochart DH, Hardinge K. Nonsurgical management of supracondylar fracture above total knee arthroplasty. Still the nineties option. J Arthroplasty 1997; 12: 830-834

8 Olerud S. Operative treatment of supracondylar-condylar fractures of the femur. Technique and results in fifteen cases. J Bone Joint Surg [Am] 1972; 54: 1015-1032

${ }^{9}$ Seinsheimer 3rd. F. Fractures of the distal femur. Clin Orthop Relat Res 1980; 153: 169 179

${ }^{10}$ Yang RS, Liu HC, Liu TK. Supracondylar fractures of the femur. J Trauma 1990; 30: 315319

${ }^{11}$ Neer 2nd CS, Grantham SA, Shelton ML. Supracondylar fracture of the adult femur. A study of one hundred and ten cases. J Bone Joint Surg [Am] 1967; 49: 591-613

12 Schatzker J, Lambert DC. Supracondylar fractures of the femur. Clin Orthop Relat Res 1979; 138: 77-83

13 Trentz O, Tscherne H, Oestern H-J. [Technique of internal fixation and results in distal femoral fractures (author's transl)]. Unfallheilkunde 1977; 80: 441-448

14 Tscherne H, Oestern H-J, Trentz O. [Long term results of the distal femoral fracture and its special problems (author's transl)]. Zentralbl Chir 1977; 102: 897-904

15 Bolhofner BR, Carmen B, Clifford P. The results of open reduction and internal fixation of distal femur fractures using a biologic (indirect) reduction technique. J Orthop Trauma 1996; 10: 372-377

16 Zlowodzki M, Bhandari M, Marek DJ et al. Operative treatment of acute distal femur fractures: systematic review of 2 comparative studies and 45 case series (1989 to 2005). [Review] [74 refs]. J Orthop Trauma 2006; 20: $366-371$ 
17 Henderson CE, Kuhl LL, Fitzpatrick DC et al. Locking plates for distal femur fractures: is there a problem with fracture healing? [Review]. J Orthop Trauma 2011; 25 (Suppl. 1): 88-114

18 Herrera DA, Kregor PJ, Cole PA et al. Treatment of acute distal femur fractures above a tota knee arthroplasty: systematic review of 415 cases (1981-2006). [Review] [35 refs]. Acta Orthopaedica 2008; 79: 22-27

19 Vallier HA, Immler W. Comparison of the 95degree angled blade plate and the locking condylar plate for the treatment of dista femoral fractures. J Orthop Trauma 2012; 26: 327-332

20 Baumgaertel F, Gotzen L. [The "biological" plate osteosynthesis in multi-fragment fractures of the para-articular femur. A prospective study]. Unfallchirurg 1994; 97: 78-84

${ }^{21}$ Farouk O, Krettek C, Miclau T et al. Minimally invasive plate osteosynthesis: does percutaneous plating disrupt femoral blood supply less than the traditional technique? J Orthop Trauma 1999; 13: 401-406

22 Kregor PJ, Stannard JA, Zlowodzki $M$ et al. Treatment of distal femur fractures using the less invasive stabilization system: surgical experience and early clinical results in 103 fractures. J Orthop Trauma 2004; 18: 509520

${ }^{23}$ Krettek C, Schandelmaier P, Miclau T et al. Transarticular joint reconstruction and indirect plate osteosynthesis for complex distal supracondylar femoral fractures. Injury 1997; 28 (Suppl. 1): A31-A41

24 Schutz M, Muller M, Krettek C et al. Minimally invasive fracture stabilization of distal femoral fractures with the LISS: a prospective multicenter study. Results of a clinical study with special emphasis on difficult cases. Injury 2001; 32 (Suppl. 3): SC48-SC54

25 Ricci WM, Loftus T, Cox C et al. Locked plates combined with minimally invasive insertion technique for the treatment of periprosthetic supracondylar femur fractures above a total knee arthroplasty. J Orthop Trauma 2006; 20: 190-196

26 Gray AC, White TO, Clutton E et al. The stress response to bilateral femoral fractures: a comparison of primary intramedullary nailing and external fixation. J Orthop Trauma 2009; 23: 90-97
Dr. med. Marie K. Reumann

Postdoctoral Research Fellow

Institute of Health and Biomedical

Innovation (IHBI)

Queensland University of Technology

(QUT)

60 Musk Avenue, Kelvin Grove

Brisbane, QLD 4059

Australia

Prof. Dr. med. Michael Schuitz, FRACS (Ortho.surg.), FAOrthA

Professor and Chair in Trauma

Institute of Health and Biomedical

Innovation (IHBI)

Queensland University of Technology

(QUT)

60 Musk Avenue, Kelvin Grove

Brisbane, QLD 4059

Australia

Director of Trauma,

Princess Alexandra Hospital

Metro South Health Service District

Trauma Service

199 Ipswich Road, Woolloongabba

Brisbane, QLD 4102

Australia

m.schuetz@qut.edu.au 